\title{
Al compás de las clases de baile: una experiencia dentro del aula
}

\author{
At the beat of dance lessons: a classroom experience
}

\author{
Cecilia Enith Romero-Barquero ${ }^{1}$ \\ Universidad de Costa Rica \\ Escuela de Educación Física y Deportes \\ San José, Costa Rica \\ Cenith14@yahoo.es
}

\section{Recibido: 11 julio 2013 Aceptado: 4 diciembre 2014 Corregido: 25 setiembre 2014}

\begin{abstract}
Resumen: Esta investigación procuró entender e interpretar el contexto educativo en el que se desenvolvió un grupo de estudiantes universitarios, en un curso de baile popular. Los objetivos del estudio se orientaron a conocer si la metodología y la labor docente mediaron en el aprendizaje y en el esparcimiento del alumnado, y en detectar si el trabajo en clase influyó en las relaciones interpersonales del estudiantado. Este estudio cualitativo usó métodos de investigación-acción y etnográficos, técnicas de observación participante, notas de campo, video, tarjetas de respuestas, reportes orales y escritos del asistente y grupo focal. En el análisis de datos se conformaron temas de interés, luego se establecieron categorías y sub-categorías en cada técnica y, finalmente, se realizó la triangulación. Los resultados mostraron que la metodología del curso promovió el esparcimiento del estudiantado y que la sensación de satisfacción y el gozo fomentaron la realización de actividad física, como el baile popular. Además, se halló que la estructura de las lecciones, los estilos de enseñanza y la labor docente influyeron positivamente en el aprendizaje de los contenidos teóricos y prácticos del curso. También, se encontró que el trabajo en clase repercutió en las relaciones interpersonales del grupo participante, por medio de la promoción de valores, normas de higiene, un ambiente ameno y la proyección del baile como una actividad socio-cultural. Los hallazgos del estudio se reportan con la intensión de que sean transferibles a otros contextos educativos, en los cuales el contenido del programa por impartir sea el baile popular.
\end{abstract}

Palabras claves: Investigación-acción, etnografía, baile popular, relaciones interpersonales, procedimientos metodológicos

Abstract: This research sought to understand and interpret the educational context in which a group of university students took part, in a popular dance course. The objectives of the study were designed to determine whether the methodology and teaching influenced the learning and leisure of the students, and to detect if the class work influenced the student's interpersonal relationships. This qual-

1 Cecilia Enith Romero Barquero: magíster en Movimiento Humano y Recreación y bachiller en la Enseñanza de la Educación Física de la Universidad de Costa Rica. Ha participado como tallerista y expositora en congresos a nivel nacional e internacional, como por ejemplo: Encuentro Internacional de Campismo, Congreso Centroamericano de Educación Física, Deportes y Recreación. Posee estudios en baile popular, natación, bádminton, fútbol y áreas educativas y recreativas. Ha colaborado como recreacionista con albergues del PANI, EBAIS, Fundación Da, Omar Dengo, Aldeas S.O.S., ICODER, UNICEF, ALCCI, entre otras instituciones, además de desarrollar proyectos en el área de recreación familiar y al aire libre. Ha coordinado el TCU-110 y ha colaborado en el proyecto de extensión docente: Aportes a la calidad educativa costarricense. Actualmente es profesora en la Universidad de Costa Rica, en la sede Rodrigo Facio, en la Escuela de Educación Física y Deportes, además, coordina el TCU-537 y cursa el doctorado en Investigación en Ciencias del Movimiento Humano. Entre sus publicaciones recientes se destaca: Propuesta organizacional del área de recreación para la Universidad Estatal a Distancia en Revista Educación. Y, Rally-salón: Una propuesta metodológica para realizar en clases de educación física y en proyectos recreativos en Revista digital de Educación Física EmásF. 
itative study used action-research and ethnographic methods, participant observation techniques, field notes, video, response cards, oral and written reports from the class assistant and focal groups. During the data analysis, topics of interest were determined, then categories and sub-categories were settled for each technique and, finally, a triangulation was performed. The results showed that the course methodology promoted students' leisure and the feeling of satisfaction and joy fomented physical activity, such as popular dance. It was also found that the teaching styles, structure of the lessons, and teacher's work positively influenced the learning of the theoretical and practical contents of the class. Also, it was found that class work took part in the interpersonal relationships of the participant group, through the promotion of values, standards of hygiene, a pleasant environment and the projection of dance as a socio-cultural activity. The findings of this study are reported in order to transfer them to other educational contexts, in which the content of the program is to teach popular dance.

Keywords: action-research, ethnography, popular dance, interpersonal relationships, and methodological procedures

\section{Introducción}

El presente estudio se planteó con la finalidad de conocer e interpretar el contexto educativo en el que se desenvolvía un grupo de estudiantes universitarios, que participaron en un curso de baile popular. Por lo tanto, se realizó una investigación con un diseño cualitativo, que contenía métodos etnográficos y de investigación-acción. El estudio tuvo dos objetivos: 1-conocer si la metodología y la labor docente influyeron en el aprendizaje y en el esparcimiento del alumnado y 2- detectar si el trabajo en clase influyó en las relaciones interpersonales del estudiantado.

En cuanto a los métodos, Sandin en el 2003 menciona que, la investigación-acción permite diagnosticar una situación, provoca la generación y aplicación de estrategias que ayudan a clarificar dicha situación, por medio de los resultados obtenidos.

Por su parte, la etnografía educativa es uno de los métodos que analiza las interacciones entre distintos grupos sociales y culturales que se hallan dentro de un marco educativo (Sandin, 2003). El grupo social en este estudio fue el estudiantado universitario que provenía de diversas carreras y que asistió al curso de baile popular, prácticamente sin conocerse. El marco educativo en el que se desenvolvió el grupo fue un curso que no tenía relación directa, en la mayoría de los casos, con el currículo de las carreras universitarias del alumnado.

El curso era parte de una lista de opciones de actividad deportiva. De ese listado, la población estudiantil escogía el curso que le gustaba o el que le servía matricular, según su horario, y concursaba por un cupo, según el promedio ponderado de notas. Para graduarse de la carrera universitaria, es obligatorio haber aprobado un curso de actividad deportiva; como parte de las asignaturas de Estudios Generales.

En el momento de matricular el curso en línea, el alumnado solo sabía el nombre del curso, el número de grupo, el número de la clase y el horario. A partir de esa información, las ideas que podían tener de las lecciones eran las deducciones que surgían por el nombre del curso o por el comentario de alguna persona ex-alumna del curso. Por lo tanto, desconocían quienes serían las personas con las cuales iban a compartir el curso, la persona docente, cómo sería la metodología, los contenidos, los objetivos o la evaluación que tendría la asignatura durante el semestre. 
Debido a las características del método etnográfico, todas las acciones que se realizaron en esta investigación, tuvieron como propósito el comprender qué pensaba la población participante acerca del curso, el describir cómo era el contexto educativo en el que se desenvolvía el curso, el explicar las situaciones observadas por medio del uso de diferentes técnicas cualitativas y, luego del análisis, el promover la transferibilidad del estudio.

\section{Contexto institucional}

El contexto en el que se desarrolló el curso y en el que se desenvolvieron las personas que participaron en la clase se describe en los siguientes apartados. Para ello, es necesario entender la realidad de los actores que intervinieron en el estudio porque, de acuerdo con Rodríguez, Villalobos y Mac Donald (2006), en el mundo, las personas se encuentran con otras que tienen perspectivas diferentes o, por el contrario, similitudes que comparten; y el lenguaje permite expresar lo que se percibe, e indica de manera subjetiva cómo se crea una experiencia en particular.

\section{Curso de actividad deportiva.}

El curso en el cual se desenvolvió esta investigación formó parte de la Cátedra de Actividad Deportiva de la Escuela de Educación Física y Deportes de la Universidad de Costa Rica. Estos cursos iniciaron gracias a la iniciativa del profesor Rodrigo Leiva, quien, en el año de 1957, planteó la propuesta a las autoridades para que se incluyera como parte de los Estudios Generales, como materia obligatoria, lo cual fue una realidad a partir de 1958. Sin embargo, al inicio, el curso no era obligatorio para toda la población estudiantil. Estaban exentas de él las personas casadas o personas con alguna discapacidad, religiosos y mayores de 35 años. Era ofrecido cada 15 días durante todo el año, pues los grupos eran grandes, de 250 personas. Luego, en 1972, se hizo obligatoria para todo el alumnado. En 1975, la frecuencia de las clases era de una vez a la semana y, en 1977, pasó a formar parte del Departamento de Educación Física y cambió a modo semestral (García, 1979).

En la actualidad se ofrecen entre 60 y 70 cursos de actividad deportiva, que se ponen a disposición del estudiantado universitario cada semestre. Los cursos son distintos entre sí y conforman una amplia variedad de opciones. Todos tienen el objetivo de estimular los canales socio afectivo, físico motor y cognitivo, mediante la participación activa en las lecciones progresivas. Además, promueven que el alumnado adquiera conocimientos y destrezas que le permita integrar en su vida la práctica de la actividad física continua, como parte del mantenimiento y el desarrollo de su salud integral (Romero, 2012).

\section{Curso de baile popular.}

Esta investigación se desarrolló en el curso de actividad deportiva: Bailes Populares Principiante Mixto. Éste poseía una duración aproximada de una hora con cincuenta minutos por cada sesión, en este caso de 11 a.m. a 12:50 p.m. Se impartió un día a la semana, durante un periodo de 17 semanas, mismo tiempo en el que se desenvolvió esta investigación. El curso era de carácter obligatorio. Se evaluó por medio de la asistencia y la participación activa. Si 
el estudiante aprobaba el curso; entonces, no podía volver a matricular otro curso de actividad deportiva. Los cursos de Baile Popular tienen como objetivos: "propiciar la cooperación y el respeto propio de la ética del baile, además de la autoconfianza y creatividad mediante la participación en las actividades colectivas y de pareja" y "favorecer el conocer, diferenciar y ejecutar diferentes ritmos populares, a la vez que la persona participante se ejercita y fortalece patrones básicos de movimiento" (Romero, 2012, p. 1).

\section{Escenario: instalación para dar el curso.}

El lugar en el que se impartieron las lecciones fue un salón multiuso, el cual era espacioso, rectangular, libre de obstáculos, con ventanas de celosías en tres costados. Tenía buena iluminación, ya que contaba con varias lámparas en el techo, el piso era de madera y en las paredes de los extremos del salón había espejos, y dos bodegas para guardar materiales deportivos. Estaba acondicionado con parlantes y planta de sonido, así como con material deportivo como bolas de hule, colchonetas, mancuernas, aros, entre otros. En general, contenía los elementos necesarios para impartir las clases de baile popular.

\section{Método}

Este estudio se realizó bajo una metodología cualitativa, que implicó métodos de carácter etnográfico, como también de investigación-acción. Ambos tipos de métodos estuvieron orientados en descubrir los acontecimientos cotidianos que ocurrían dentro del ámbito educativo de una clase de baile popular, en un grupo de estudiantes universitarios.

La investigación-acción, según Sandin (2003), contempla cuatro etapas. Primero, se clarifica y diagnostica una situación problemática para la práctica, que en este caso se hizo por medio de observaciones sistemáticas por parte de la profesora-investigadora en el aula y de grupos focales. El problema que se pretendía clarificar era si la metodología y el actuar de docentes y del alumnado influían en el aprendizaje y en las relaciones interpersonales.

Segundo, se formulan estrategias con el propósito de actuar para solucionar el problema. Para ello, en este estudio las estrategias que se tomaron fueron las decisiones prácticas acerca de qué se debía hacer, quién, dónde y cuándo. Por lo tanto, se tomó la decisión de realizar observaciones, tomar notas, tener en cuenta un reporte escrito y otro oral del asistente, al finalizar cada lección, y pasar una tarjeta de respuesta, en una de las clases, para que el alumnado escribiera, de manera anónima, su opinión acerca del curso. Por ejemplo: cómo se desarrollaba el curso, si les gustaba y porqué, qué le parecían las actividades y, a la vez, cuál era su criterio acerca del aprendizaje logrado hasta ese día de clases, según los contenidos del curso. Luego, se tomaron varios videos para analizar las reacciones, los comentarios, los gestos y las acciones del estudiantado durante la lección, del asistente de la clase y de la profesora. Por último, se planeó un grupo focal con los temas y categorías que se habían obtenido de las técnicas descritas anteriormente, con el propósito de interpretar mejor las relaciones interpersonales entre estudiantes y la eficacia de los procedimientos metodológicos usados en clase y la labor docente. Todas estas estrategias fueron planeadas por la profesora-investigadora del curso. 
El tercer paso propuesto por Sandin (2003) pone en práctica las estrategias y se evalúan para comprobar una hipótesis. Por lo tanto, todas las estrategias descritas anteriormente se aplicaron para comprobar las siguientes hipótesis: 1- los procedimientos metodológicos que se llevaron a cabo y la labor de la profesora y del asistente en el curso influyeron en el aprendizaje y en el esparcimiento del alumnado y 2- las relaciones interpersonales entre la población estudiantil eran agradables y facilitadas, gracias al trabajo en clase.

Siguiendo el proceso de investigación-acción, discutido por Sandin (2003), la última etapa revela una nueva aclaración y diagnóstico de la situación problemática, con base en los resultados obtenidos, lo cual provoca una nueva reflexión y acción. Estos resultados son presentados por medio de un informe que, en este caso, viene a ser este artículo.

Por su parte, la etnografía, de acuerdo con Erickson, citado por Sandin (2003), es un proceso de investigación por el cual se aprende un modo de vida de algún grupo, en este estudio fue del grupo de estudiantes del curso de Baile Popular. Además, Goetz y LeCompte (1988, p. 41) consideran que la etnografía educativa aporta "valiosos datos descriptivos de los contextos, actividades y creencias de los participantes en los escenarios educativos". Estas autoras plantean cuatro fases. La primera consiste en establecer las preguntas de la investigación y seleccionar al grupo. Segundo, la persona investigadora entra al escenario y se determina la población informante, las fuentes de datos y se establecen las estrategias. Tercero, se realiza el trabajo de campo. Cuarto, se efectúa el análisis de la información. Estas fases se aplicaron en esta investigación.

\section{Objetivos del estudio}

1. Conocer si la metodología y la labor docente influyeron en el aprendizaje y en el esparcimiento del alumnado.

2. Detectar si el trabajo en clase influyó en las relaciones interpersonales del estudiantado.

\section{Participantes}

Las personas del estudio fueron seleccionadas de manera intencional, de acuerdo con la matrícula en el curso de Baile Popular Principiante Mixto. En total, fueron 17 hombres y 17 mujeres, con edades entre 17 a 27 años, con mucho, poco o ningún conocimiento de baile popular. En general, eran personas que no se conocían hasta el primer día de clases, en el cual se realizaron algunas actividades de integración. Para el ciclo lectivo, el curso contó con un asistente, quien cursaba el último año de la carrera de la Enseñanza de la Educación Física, hacía su práctica docente y se encargaba de algunas partes de la lección de baile popular.

\section{Técnicas de recolección de datos}

Para la recolección de los datos se utilizaron las siguientes técnicas cualitativas etnográficas y de investigación-acción. 


\section{Observación participante.}

La técnica llamada observación participante resulta ser, según Artavia (2011), "una técnica muy importante para recoger datos, pues permite al investigador pasar gran cantidad de tiempo en contacto directo con los sujetos en estudio y así, vivir de manera cotidiana las interacciones y actividades que estos ejecutan" (p. 200).

Como parte de los procesos de la investigación-acción, en este estudio, la investigadoradocente, al iniciar el curso, realizó primero una observación del lugar en el que se realizaban las lecciones. Los aspectos observados de la instalación y materiales se anotaron en una bitácora con el propósito de analizar, si el espacio físico en el cual se desenvolvía el estudiantado durante el curso, facilitaba la práctica de baile popular.

Luego, durante cada lección la investigadora realizó observaciones del comportamiento y accionar de todas las personas involucradas en la clase. Estas observaciones fueron apuntadas en las notas de campo, las cuales se complementaron con otras técnicas como reporte escrito y oral del asistente de clase, tarjetas de respuestas, grupo focal y técnicas de documentación.

\section{Reporte escrito y oral del asistente de clase.}

El asistente estaba a cargo de ciertas secciones de la clase, como parte de las asignaciones del curso de práctica docente. Por lo tanto, él dirigía varias actividades y, a la vez, ayudaba en la corrección de las destrezas y postura del alumnado. Sus acciones estuvieron orientadas por la profesora del curso de baile popular, quien le asignaba tareas o responsabilidades, con el propósito de que él se enfrentara a la realidad de estar a cargo de un grupo, pusiera en práctica sus conocimientos y ganara experiencia. Al final de cada lección, el asistente informó a la profesora-investigadora sus vivencias y aprendizajes por medio del reporte escrito y el oral. Además de indicar las apreciaciones personales acerca de su práctica docente, el asistente ofreció un reporte acerca de las observaciones hechas al grupo de estudiantes, con respecto al comportamiento y actitudes que éste mostraba durante la lección. Esta información fue analizada luego por la investigadora con el fin de compararla con las observaciones hechas por ella, así como con los videos y las tarjetas de respuestas del estudiantado.

\section{Tarjetas de respuestas.}

Con esta técnica, se realizaron algunas preguntas concretas acerca de un tema específico. La población estudiantil del curso anotó sus respuestas en una tarjeta de $11 \mathrm{~cm}$ de ancho por $14 \mathrm{~cm}$ de largo (un cuarto de hoja de papel). Esta técnica permite que las personas participantes opinen de manera concreta y respondan sin angustiarse por tener que escribir en un espacio grande. En ocasiones, las personas escriben mucho para que la hoja no se vea muy vacía (Guillermo Alfaro, comunicación personal, 21 agosto 2012).

En este estudio, el uso de la tarjeta se empleó en la cuarta clase. La pregunta que se hizo al estudiantado correspondía a su opinión personal acerca del curso. De acuerdo con las respuestas escritas por el alumnado, se generaron tres temas de interés: 1- labor de la profesora y el asistente, 2- metodología del curso y 3- relaciones interpersonales entre el 
alumnado. Estas categorías se compararon con las notas de campo, videos y los reportes escritos y orales del asistente.

\section{Grupo focal.}

Según, Artavia (2011), el grupo focal consiste en:

Entrevistas de grupo, donde existe una dependencia de la interacción dentro del grupo, basada en los temas o preguntas formuladas por el investigador. El investigador plantea una pregunta o grupo de preguntas articuladas, que versan sobre el fenómeno en estudio, las cuales deben ir siendo reorientadas en la búsqueda de la información precisa. (p. 204)

En esta investigación se realizó un grupo focal al inicio y otro al final del curso. En ambas ocasiones se reunió a toda la población estudiantil que estuvo presente ese día de lecciones, además del asistente de clase. La conversación fue moderada por la investigadora, quien usó una guía de preguntas generadoras para orientar la discusión. Los comentarios que se dieron durante el grupo focal fueron grabados por medio de video. Además, la investigadora tomó nota de las opiniones e ideas que se fueron brindando, las cuales fueron comparadas posteriormente con el video y las demás técnicas.

El primer grupo focal fue utilizado como diagnóstico por la profesora-investigadora. Las preguntas iban orientadas a conocer las expectativas que tenía el alumnado con respecto al curso, así como para analizar el grado de experiencia y conocimientos que poseía el estudiantado acerca de los diferentes ritmos de baile popular y su grado de participación en actividades físicas y de ejercicio.

En el segundo grupo focal, las preguntas fueron dirigidas a confirmar o descartar aspectos obtenidos por medio de las observaciones, videos de clase, reportes orales y escritos del asistente y las tarjetas de respuestas.

\section{Técnicas de documentación.}

Otra técnica utilizada en el estudio fue la videograbación, la cual permite obtener información confirmatoria con respecto a los datos obtenidos por medio de varias técnicas cualitativas, como observaciones y grupos focales. Al respecto, Reyes (2005) considera que la grabación de videos permite captar palabras, acciones y el ambiente que se vive en los espacios reales.

En este estudio, los videos fueron realizados por la investigadora en cinco ocasiones. Dos de ellas, durante los grupos focales, y las otras tres en las clases número 3, 8 y 13, de manera que abarcara el inicio, el medio y casi el final del curso.

\section{Análisis de datos}

En las investigaciones cualitativas, el análisis de datos debe realizarse de manera continua. Por tanto, en este estudio se analizaron y codificaron ininterrumpidamente los datos obtenidos, por medio de las distintas técnicas usadas: observaciones, videos, tarjetas de 
respuestas, reportes orales y escritos del asistente y grupos focales (Artavia, 2011). Molina (2009) considera que "el muestreo, la recolección y el análisis de datos resultan actividades casi paralelas, por lo que el análisis cualitativo implica reflexionar constantemente sobre los datos recogidos, organizarlos y transcribirlos" (p. 53).

Entre los pasos realizados en este estudio, primero se analizaron las notas de campo de cada observación de las lecciones, así como de los videos que se efectuaron. Con el fin de detectar comentarios, gestos y acciones que tenía el estudiantado, mientras participaba en la lección, además del comportamiento y reacciones que tenía el asistente cuando impartía o colaboraba en la gestión de la clase.

A la vez que se analizaban los datos, se fueron haciendo categorías de los aspectos que se repetían más constantemente en las notas de las observaciones y videos. Este procedimiento se mantuvo durante todos los días de clase. Según Artavia (2011), "la categorización permite examinar los datos con la intención de identificar aquellas unidades que posibiliten su clasificación, en una u otra categoría de contenido" (p. 213).

Además de las observaciones, se analizaron los reportes escritos y orales que el asistente elaboró después de cada una de las clases. También, se procedió en la cuarta clase a repartir una tarjeta, en la cual cada estudiante, anónimamente, escribió su criterio con respecto al curso. Las respuestas obtenidas en estas tarjetas, así como la información de las notas de campo, videos y reportes del asistente, ratificaron tres áreas de interés vinculadas con la actitud del estudiantado hacia: 1- los procesos metodológicos del curso, 2- la profesora y el asistente y 3- las relaciones interpersonales entre estudiantes.

A partir de estas áreas, la información se categorizó y subcategorizó. Para ello, se estableció un color para cada categoría y un símbolo para cada subcategoría. En la Tabla 1 se muestra un ejemplo de este proceso de codificación.

\section{Tabla 1}

Ejemplo de categorización y simbolismo

\begin{tabular}{lllll}
\hline Área de interés & Categoría & Color & Subcategoría & Símbolo \\
& & & & \\
\hline Actitud del & Relaciones & & Ambiente ameno & $-\cdots---$ \\
estudiantado & interpersonales & & Promoción de valores (compañerismo, & - \\
hacia las & entre & Azul & ayuda mutua). & \\
relaciones & estudiantes & & Baile como actividad socio-cultural & \\
$\begin{array}{l}\text { interpersonales } \\
\text { entre compañeros }\end{array}$ & de baile popular & & Normas de higiene & $* * * * *$ \\
\hline
\end{tabular}

Nota: Elaboración propia 
Además, la información que se recabó con las notas de campo, videos, reportes y tarjetas de respuestas, se replanteó en una serie de preguntas del grupo focal de mediados del curso. Esta actividad se desarrolló con el propósito de confirmar con la población estudiantil si la interpretación de los datos recabados, hasta el momento, correspondía con la realidad del alumnado.

Para finalizar el análisis de los datos, se realizó una triangulación para validar los resultados obtenidos tanto con las observaciones directas y de video, así como con las tarjetas de respuestas, los reportes orales y escritos del asistente y los grupos focales. Según Colás y Buendía (1994), la triangulación de fuentes comprueba si las informaciones aportadas por una fuente son confirmadas por otra.

\section{Resultados y discusión}

Los resultados de la información obtenida por medio de las distintas técnicas, con respecto a la metodología de clase, a la labor de la profesora y del asistente, y a las relaciones interpersonales entre el estudiantado, se presentan en la Figura 1. Estas tres categorías se discutirán en esta sección.

\section{Importancia de la metodología del curso en el esparcimiento del alumnado}

\section{- Promoción del esparcimiento por medio del curso.}

El reconocer los factores que influyen en el estudiantado es substancial para que este grupo participe en las clases y a la vez disfrute haciéndolo, aun cuando sea una actividad obligatoria, como son los cursos de actividad deportiva. Durante el análisis de los datos se determinó que la población estudiantil participaba en las actividades y que además disfrutaba el curso y se sentía bien durante las lecciones. Este actuar se relaciona con el concepto de esparcimiento. Según Salazar (2007), esparcimiento es aquella sensación de gozo y satisfacción que se genera en las personas cuando realiza alguna actividad de su agrado.

El análisis de las observaciones y videos confirmaron que al alumnado le interesó y disfrutó el curso porque ponía atención a las instrucciones, practicaba los pasos, se ayudaba entre sí, se entusiasmaba (aplaudía, gritaba de alegría, de vez en cuando) y, al final de la clase, aplaudía por el trabajo realizado. 


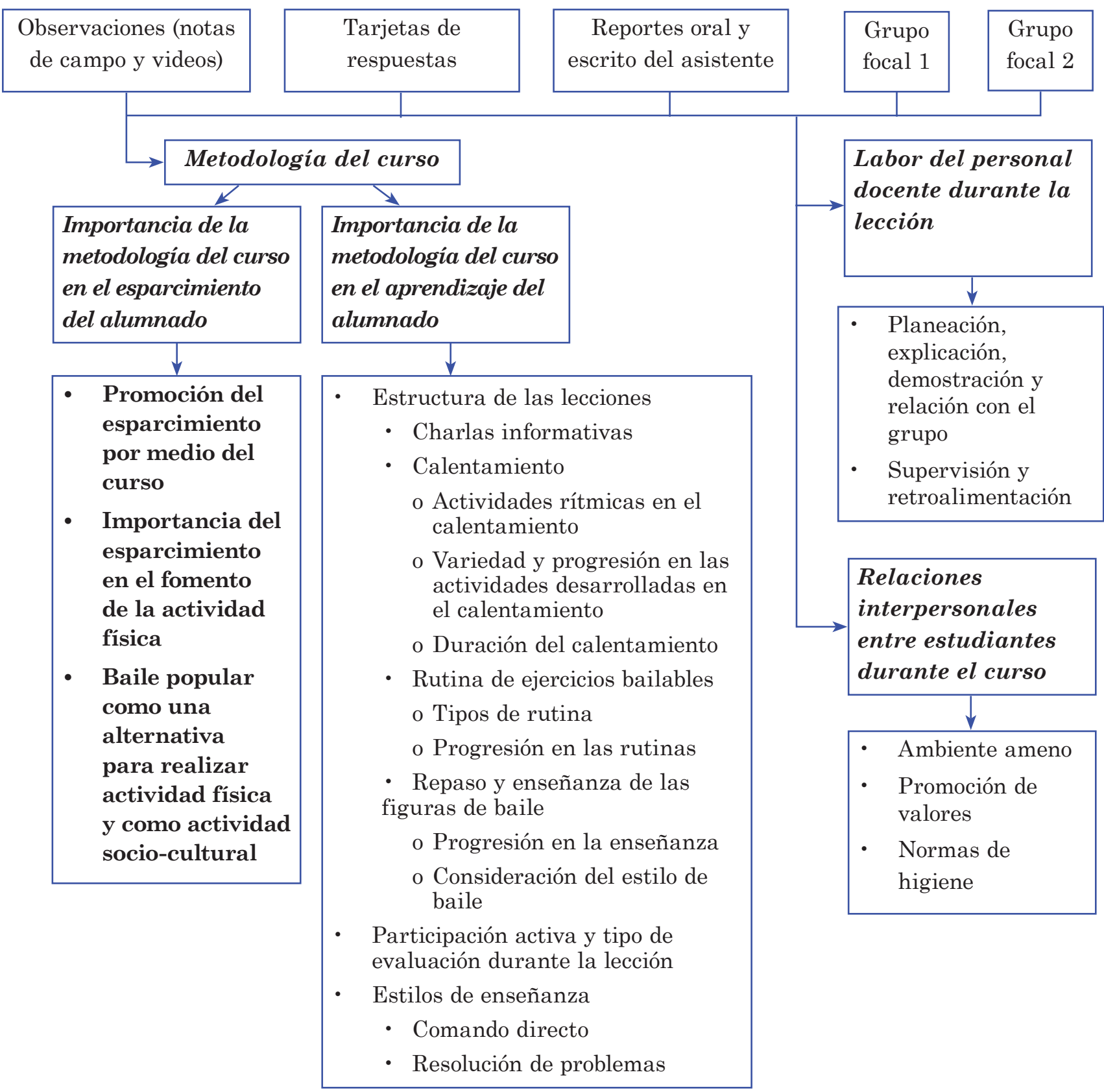

Figura 1. Triangulación de resultados obtenidos con las técnicas de documentación

Nota: Elaboración propia

Además, en las tarjetas de respuestas, algunos estudiantes indicaron, "me parece muy bueno el curso, los profes exc! Explican muy bien los pasos y las clases son entretenidas. No aburren y dan ganas de venir. Sí se aprende a bailar!", "he aprendido bastante, me gusta muchisimo y que dicha que todavía no se acaba el semestre", "el curso es el que más me motiva del semestre". 
El hecho de que el curso le pareciera interesante, provechoso y ameno al estudiantado, no sólo se evidenció por medio de los comentarios y las anotaciones de la población estudiantil, sino también por el uso de dibujos. Por ejemplo, entre las notas que escribió el alumnado en las tarjetas de respuestas se hallaron unas muestras pictográficas (caritas felices) que culminaban el comentario de cinco personas. Además, ninguno de los papeles tenía un comentario negativo que indicara molestia o desagrado por la clase.

\section{- Importancia del esparcimiento en el fomento de la actividad física.}

Aunque el curso de Baile Popular pertenece a la educación formal (carácter obligatorio) y no tiene relación directa con el currículo de las carreras universitarias de la mayoría de la población estudiantil, se logró cautivar al alumnado de manera que disfrutara las actividades que realizaba durante la clase y lo motivó a seguir practicándolas. Este logro es importante ya que, de acuerdo con Castillo, Alvarado y Sánchez (2006), en las lecciones de Educación Física, a pesar de ser importantes para la promoción de la actividad física, la gente pierde el gusto por su práctica. Esta situación es producto de que las clases son vistas por la población estudiantil como una obligación que genera apatía, en especial, en aquellas personas que no poseen una adecuada condición física para cumplir con las actividades.

El ser poco activo físicamente fue una característica que también se encontró en el grupo de estudio. Por ejemplo, en el primer grupo focal, realizado el primer día de clases, se consultó al estudiantado si solía realizar algún tipo de actividad física, considerando ésta como "cualquier movimiento corporal producido por los músculos esqueléticos que provocan un gasto energético, por encima de los niveles de reposo basal" (Garber et al. 2011, p.1337). Gran parte del estudiantado coincidió en que no acostumbraba realizar actividad física, ni tampoco ejercicios. En cuanto a la práctica de baile popular, la respuesta fue similar. La mayoría manifestó tener poca experiencia y que no lo practicaba. Solo dos estudiantes señalaron que sabían bailar varios ritmos.

Con el uso de las tarjetas de respuestas se encontraron comentarios que indicaron que el curso se convirtió en un espacio único, que permitió la práctica de actividad física, por medio del baile popular. Al respecto, un estudiante señaló, "me siento muy bien, es la única oportunidad que tengo de bailar con otras personas a la semana".

Además, luego del análisis del video del primer grupo focal, se destacó el comentario que realizó la profesora del curso, quien indicó, "es constante que el alumnado no saque tiempo para realizar actividad física. De ahi la importancia de este tipo de curso, porque además de enseñar una determinada destreza, es la oportunidad para enseñar aspectos importantes acerca de la salud y hacer conciencia de lo necesario que es realizar actividad física constante". Según Romero (2009b), una de las principales barreras que alega la población estudiantil para no practicar actividad física, incluso baile popular, es la falta de tiempo. Sin embargo, de acuerdo con comentarios de la docente, en el primer grupo focal, y según los objetivos de los cursos de actividad deportiva, se pretende motivar al estudiante para que una vez terminado el curso organice su tiempo para que pueda realizar actividad física constante. 


\section{- Baile popular como una alternativa para realizar actividad física y como actividad socio-cultural.}

Considerando la información analizada, se notó como al estudiantado le gustó el curso de baile popular, disfrutó realizar las actividades que se desarrollaban en la clase y, a medida que pasaron las lecciones, adquirieron y mejoraron las destrezas requeridas para bailar. Por consiguiente, el baile popular se convirtió en una alternativa para que, al finalizar el curso, el estudiantado siguiera realizando actividad física.

Un ejemplo obtenido de las notas de campo, que reflejaba que la población estudiantil estaba alegre y participativa al realizar las actividades físicas durante la clase, fue cuando el asistente realizó un calentamiento. En esta ocasión, el alumnado tenía que estar bailando en pareja. Una alumna quedó sin compañero y tenía que esperar turno durante la dinámica. Otra de las estudiantes tenía que salir del juego para dar lugar a la que esperaba turno. Pero cuando se pidió que alguna de las mujeres saliera, las estudiantes dudaron unos segundos en tomar la decisión de salir. Para la profesora, esta reacción "es de destacar, porque cuando una actividad no se disfruta, las personas no dudan en salir de la actividad a la primera oportunidad que tengan y, en este caso, fue todo lo contrario. A pesar de ser una actividad física y que requería de constante movimiento, los chicos se veían que lo estaban disfrutando".

$\mathrm{El}$ agrado del alumnado por el curso de baile popular, también se demostró por medio de dos estudiantes, que en diferentes oportunidades se acercaron a la profesora para indicarle que se sentían un poco enfermos y le avisaban por si tenían que sentarse un rato, o realizar las actividades a menor intensidad. Esta solicitud es permitida, a pesar de que la participación debe ser cien por ciento activa durante el curso para no quedar ausentes, pero esto no aplica a quienes piden permiso para descansar porque no se sienten bien por algún malestar o cansancio. Sin embargo, y de acuerdo con lo observado en la lección, en ningún momento los alumnos que manifestaron sentirse mal, al inicio de la clase, se sentaron o dejaron de realizar las actividades. Por el contrario, permanecieron alegres y participaron en toda la lección. Al respecto, Valverde (2002) señala que por medio de la danza se pueden obtener muchos beneficios como aumentar las endorfinas que producen sensación de bienestar y mejorar la condición física, entre otros.

Por otra parte, el gusto por el curso de baile popular también se manifestó en comentarios realizados por el alumnado, durante el segundo grupo focal. Por ejemplo, un estudiante señaló: "nos gusta el baile, aunque no seamos muy buenos, pero queremos aprender". La profesora manifestó, "el hecho que a la gente le guste la clase, lo veo mucho en los cursos de baile, a diferencia de otros cursos en los cuales a veces los estudiantes matriculan porque no tienen otra opción de horario, debido a que los demás cursos no se ajustan a su horario y tienen que matricular algo". Además, agregó, "de acuerdo con encuestas que he pasado en otras ocasiones, la mayoría de las personas, con excepción de tal vez uno o dos casos, matriculan el curso de bailes porque les gusta o quieren aprender".

Otro ejemplo que respalda los comentarios anteriores es el comportamiento observado en el alumnado. Antes de iniciar la clase o durante las actividades pasivas, la población estudiantil se comportó callada, calmada, incluso, en algunos casos, hasta tímida, pero bastaba con que sonora la primera pieza para que las personas se vieran más contentas, 
sonrientes, conversadoras y participativas. La profesora opinó en el primer grupo focal, "la gente hace ejercicio con el baile y no lo siente. Se le pasa el tiempo rápido, porque lo está disfrutando". García (1979) considera importante que el alumnado se sienta libre, entregado a la actividad deportiva, porque esto le permite mayor satisfacción y armonía.

Es importante recalcar que el gusto por el baile también mostró valor cultural. Por ejemplo, durante el primer grupo focal, la profesora mencionó que el baile tiene relación con la cultura, al decir, "el baile es parte de la cultura de nuestro país, incluso de nuestra etnia como latinos. Es algo que nos caracteriza, lo cual hace que nos guste, o en otros casos que nos sintamos comprometidos en aprender. Porque el baile no solo nos sirve para hacer ejercicio, sino también para socializar. En casi todas las fiestas hay música y la gente suele bailar y si no sabes; entonces, eso se convierte en un espacio menos en el que puedes participar y compartir con otros". Al respecto, Bennett, citado por Zavatto y Gabbei (2008), indica que la enseñanza culturalmente sensible incluye escogencia de contenidos (en este caso tipos de baile) que la población estudiantil reconoce de su cultura. Además, Morera et al., citados por Garnier (2009), señalan que, en los programas de educación física, la música puede provocar sentimientos en el alumnado que son expresados con movimientos y, por lo tanto, el personal docente debe preparar actividades que permitan dicha expresión, además de la transferencia a espacios de la vida cotidiana del estudiantado.

Con respecto a los aportes del baile, Valverde (2002) indica que la práctica del baile permite descubrir habilidades corporales, promover el deseo de auto superación, estimular la resolución de problemas, incrementar la creatividad, la autoconfianza, la motivación, las relaciones interpersonales y disminuir actitudes de indecisión. Estos aportes del baile colaboran para que la persona que participa en las clases se sienta bien, emocionada, contenta porque está aprendiendo y mejorando. Algunas de las personas participantes en el curso indicaron en las tarjetas de respuestas: "me parece un curso entretenido donde se disfruta y aprende al mismo tiempo, tanto como para los que ya saben bailar como a los principiantes, es muy provechoso". Otro estudiante señaló, "me siento muy bien en el curso, he aprendido demasiado a limpiar los movimientos". Otra persona comentó, "Ime parece excelentej he aprendido mucho, lástima que no hay más horas de lección".

El cambiar hábitos es difícil, pero posible. Por lo que cabe la posibilidad de fomentar la práctica de la actividad física constante por medio del baile. Al respecto, se rescatan dos comentarios de estudiantes que no acostumbraban realizar actividad física y que, a partir de la práctica en el curso, cambiaron su visión. Uno de ellos señaló, por medio de la tarjeta de respuestas, "las clases son entretenidas, no aburren y dan ganas de venir, sí se aprende a bailar". El otro mencionó, "me gustaría de hecho seguir llevándolo y aprender más".

\section{Importancia de la metodología del curso en el aprendizaje del alumnado}

Como se mencionó anteriormente, el hecho de que el curso fuera del agrado del estudiantado fue muy beneficioso, porque generó una mayor disposición para recibir las clases y permitió crear bases para que en el futuro se desee seguir con la práctica de las destrezas aprendidas, pero como una actividad recreativa. Según Salazar (2007), las actividades 
recreativas son todas aquellas que se realizan en el tiempo libre, generan desarrollo integral en quienes las practican y se seleccionan y participa en ellas de manera voluntaria.

Tomando en cuenta estos beneficios, se debe analizar cuáles son los factores que, desde la perspectiva del estudiante, provocan que el curso sea agradable, entretenido y provechoso para el aprendizaje. De la información obtenida por medio de las distintas técnicas, y de acuerdo con la categorización realizada, se distinguen dos aspectos importantes de mencionar. El primero se relaciona con la estructura de la lección y el segundo, con la labor del personal docente, en este caso de la profesora y del asistente.

\section{- Estructura de las lecciones del curso de baile popular.}

A continuación se presentan los resultados de cómo la profesora estructuró la lección, así como los estilos de enseñanza que utilizó y cómo la población estudiantil percibió dicho planeamiento.

Por medio de las observaciones se encontró que la estructura de las lecciones era la siguiente: (1) se pasaba lista de asistencia, (2) en algunas ocasiones, se daban charlas de información acerca de la importancia y los tipos de actividad física, o algún otro tema relacionado, (3) se procedía a realizar un calentamiento de unos 20 minutos, (4) se continuaba con una pequeña rutina de ejercicios bailables, (5) se repasaban las vueltas y pasos enseñados en la clase anterior, (6) se proseguía con la enseñanza de nuevas figuras y pasos de baile y (7) se culminaba con un agradecimiento y despedida de la profesora hacia el estudiantado.

En los siguientes párrafos, se detallan los resultados encontrados por medio de las diferentes técnicas, en cuanto a cómo influyó la estructura de la lección, antes mencionada, en el aprendizaje del alumnado.

\section{- Charlas informativas.}

De acuerdo con las notas de campo y el reporte del asistente, se encontró que las charlas eran de interés del estudiantado, porque en la mayoría de éstas se realizó una dinámica interactiva entre docente, estudiantes y asistente. Este interés del estudiantado por las charlas se reflejó también en algunos comentarios escritos en la tarjeta de respuestas. Por ejemplo, un alumno indicó, "el curso me ha parecido SUPER cool!, me gustan mucho las charlas informativas y he aprendido muchisimo, sigan así chicos los felicito”. Además, acerca de las charlas, no hubo ningún comentario que se considerara negativo o de disconformidad, tanto durante ellas como tampoco en las tarjetas de respuestas.

\section{- Calentamiento.}

Con base en las observaciones y los videos, el calentamiento fue dirigido por la profesora o por el asistente. El objetivo que tenía era preparar físicamente al estudiantado para las actividades posteriores que se desarrollarían durante la clase, por medio de diferentes tipos de dinámicas. De esa forma, se incrementó progresivamente la capacidad de trabajo, ya que después de un periodo de inactividad, la falta de tonicidad, la apatía y la pereza se hacen presentes (Muñoz, 2009). Los calentamientos tienen el objetivo de "prever la aparición de lesiones y preparar física, fisiológica y psicológicamente al sujeto para la actividad de la parte principal o la competición" (p. 1). 
Otro propósito que tenía la profesora con el calentamiento era que éste sirviera como una actividad en la que el alumnado practicara el uso del ritmo del baile por aprender. Por tanto, el calentamiento fue una parte en la lección que se prestó para hacer varios análisis, de los cuales surgen los siguientes resultados.

\section{o Actividades rítmicas en el calentamiento.}

Según las notas de campo, los videos y los reportes del asistente, se contempló como el calentamiento consistió en diferentes tipos de actividades rítmicas. Por ejemplo, se implementaron cantos jocosos, juegos rítmicos, gimnasia formativa, acrosport, sistema natural con movimientos que involucraban pasos de baile, actividades de comandos, dinámicas en el piso, rutinas de seguimiento, ejercicios bailables, entre otros.

Las actividades rítmicas usadas en el curso de baile popular por la profesora y el asistente durante los calentamientos coincidieron con lo estipulado por Quiñónez (2006). Esta autora menciona que existen diversos tipos de actividades rítmicas, entre ellas: bailes folclóricos, juegos de expresión, danzas, bailes sencillos individuales y grupales, gimnasia rítmica, juegos asociados con elementos rítmicos, entre otros.

Entre las actividades rítmicas usadas en el curso estuvieron los cantos jocosos. Al respecto, Bernal y Calvo (2000) señalan que la canción conlleva la educación del oído, del sentido rítmico, de la voz, del movimiento, del gesto y, por ende, de la expresión corporal. Estos autores agregan que, el cancionero es un instrumento importante para el desarrollo de la capacidad expresiva y psico-motriz; a la vez que fomenta la interdisciplinariedad (música, lenguaje oral, dramatización, educación física) con el propio acervo cultural.

Por otra parte, el hecho de que en los calentamientos se realizaran actividades rítmicas ayudaba a que el estudiantado desarrollara la conciencia del ritmo, lo cual según la profesora, es esencial en un curso de baile popular. Algunos de los comentarios que la docente realizó durante el primer grupo focal incluyeron: "el aprender a reconocer el ritmo de los diferentes géneros musicales es muy importante, porque a veces ocurre que se pone música de salsa y la gente se pone a bailar merengue". Este comentario causó risas y asentamientos de aprobación con la cabeza, de parte de algunas personas del grupo estudiantil. La docente también manifestó, "aprender a guiarse por el ritmo de la música es necesario porque permite moverse al compás de la música”. Además, agregó, "a veces la gente sabe muchos pasos y vueltas, pero cuando usted los ve moverse no van con la música, van más rápido o más lento".

El ritmo, según Chacón (2003), "es el elemento vital generador de precisión, que ayuda a la acción motora" (p. 3). Además, Romero (2009a) indica que entre los beneficios del movimiento rítmico está desarrollar capacidades motrices, así como alcanzar conciencia corporal como instrumento musical y de expresión. Desde la antigüedad se enaltece el papel de la música como herramienta educativa. Por ejemplo, Platón indica que la música es el arte educador por excelencia. Por lo tanto, la primera vinculación formativa entre el movimiento y el ritmo se encuentra en el pensamiento griego, donde dicho filósofo afirmaba que la gimnasia y la música debían estar unidas en el proceso educativo (Fubini, 1998). 


\section{- Variedady progresión en las actividadesdesarrolladasenel calentamiento.}

En los calentamientos también se observó como las actividades desarrolladas fueron cambiando e incorporando un mayor grado de complejidad y similitud con los géneros musicales que se estaban enseñando. Este dato se analizó también por medio de los grupos focales y las tarjetas de respuestas. Por ejemplo, un comentario de la docente en el segundo grupo focal señalaba que: "en las primeras clases, el calentamiento se relaciona más con actividades rítmicas, para fomentar el ritmo y patrones básicos. Y poco a poco, se agregan más movimientos relacionados con pasos y vueltas específicas de baile”. Un estudiante, por medio de la tarjeta de respuestas, consideró que "está muy bien dividido entre calentamiento y baile, las explicaciones muy bien también”. Al respecto, Chacón y Hernández (2004) señalan que es importante que el calentamiento sea variado durante las diferentes lecciones.

\section{o Duración del calentamiento.}

Otro aspecto analizado, en cuanto al calentamiento, fue la duración. Algunas personas del alumnado indicaron, en la tarjeta de respuestas, que el calentamiento se extendía mucho. Sin embargo, durante el segundo grupo focal la profesora les explicó la necesidad de invertir aproximadamente 20 minutos en el calentamiento. Según Chacón y Hernández (2004), en las lecciones es importante realizar un calentamiento. Gremion (2005) menciona que un calentamiento que incluya ejercicio cardio-respiratorio y flexibilidad posee beneficios en actividades como el baile. Muñoz (2009) considera que un calentamiento puede durar entre 15 y 30 minutos, dependiendo de la condición de la persona, de las condiciones ambientales y del tipo de trabajo que posteriormente se realice.

Es probable que algunos estudiantes percibieran que el calentamiento era muy largo, por la ansiedad de practicar los géneros musicales, debido al gusto por el baile. Sin embargo, y teniendo en cuenta estos resultados, es necesario que el personal docente aclare qué es lo que se pretende lograr con cada actividad que se ejecuta en el calentamiento y que refuerce y explique constantemente la importancia de él. Tal y como menciona Rubio (s.f.), el objetivo del calentamiento es mejorar el sistema cardio-respiratorio, la contracción muscular, el sistema capsular, el ligamentoso, el cartilaginoso y el nervioso, así como el estado psicológico de las personas participantes.

\section{- Rutina de ejercicios bailables.}

En este apartado se destacan dos aspectos, primero los diferentes tipos de rutina utilizados en el curso y segundo, la progresión en las rutinas de ejercicios bailables.

\section{o Tipos de rutina de ejercicios bailables.}

De acuerdo con lo analizado en las notas de campo, videos y reportes del asistente, en el curso se utilizaron diferentes tipos de rutina de ejercicios bailables. Por ejemplo, se implementó el sistema de "usar y tirar", que, según Martín (1999), consiste en que la persona instructora explica un paso, se practica y luego se cambia por otro; por lo tanto, no lleva a 
un producto final. Es el modelo más básico y sencillo. También, se usó el sistema de "suma", en el que se agregan acciones motrices o estructuras (pasos) a la rutina. Es decir, se ejecuta, repite y aprende una estructura y se continúa con otra que será sumada a la primera (Rial, 2012). Por último, se practicó el programa de ZUMBA, el cual usa la música para asignar movimientos. Es decir, para cada sección musical (introducción, estrofa, coro, puente musical, etc.), se establece un determinado movimiento (Pérez, Robinson y Herlong, 2010). La mayoría de los sistemas utilizados en las rutinas del curso implementaron la composición dentro de la enseñanza del baile, que consiste en la secuencia de movimientos que se repiten en un orden establecido (Fernández, Ros y Vera, 1998).

\section{o Progresión en las rutinas de ejercicios bailables.}

Por otro lado, y de acuerdo con el análisis de los resultados de las observaciones, se encontró que la población estudiantil logró realizar las diferentes rutinas, las cuales iban requiriendo paulatinamente un mayor grado de complejidad y rendimiento físico. Por ejemplo, en la última rutina dirigida por la profesora, el grado de exigencia física, de coordinación y memoria era alto, comparado con las otras rutinas realizadas anteriormente.

$\mathrm{Al}$ analizar los movimientos de la población estudiantil en las primeras rutinas con respecto a las últimas, se detectó cómo las personas participantes realizaron los pasos indicados por la persona instructora con mayor precisión y fluidez durante la rutina, a medida que avanzaban las lecciones. De acuerdo con la profesora del curso, "es importante que el grado de exigencia vaya aumentando, porque eso le permite al estudiante mejorar su condición física, desarrollar más las destrezas, el ritmo, la coordinación, la conciencia espacial y corporal, además de adquirir nuevos pasos de baile".

Estos hallazgos reflejan cómo la profesora consideró que el variar y agregar complejidad a las rutinas, permitía que el alumnado mejorara su técnica. Este comentario coincide con Rial (2012), quien menciona que dentro del proceso de progresión que se debe seguir en las actividades que cuentan con soporte musical, se debe usar la sustitución (cambio de un paso por otro a partir del más básico), así como la variación (obtención de una versión diferente de un paso, por medio de la modificación del ritmo, las repeticiones, los niveles, planos, desplazamientos, entre otros aspectos).

\section{- Repaso y enseñanza de las figuras de baile.}

Siguiendo con la estructura metodológica de la clase, después de las rutinas de ejercicios bailables, se continuaba con el repaso o la enseñanza de las figuras de baile (pasos y vueltas). De acuerdo con los hallazgos, se rescatan dos factores importantes por tener en cuenta para impartir lecciones en un futuro, tales como la progresión en la enseñanza y el estilo de baile.

\section{o Progresión en la enseñanza.}

De acuerdo con la información analizada, se halló que las diferentes figuras y pasos fueron enseñados de manera progresiva, del más sencillo al más difícil. Un estudiante 
escribió en la tarjeta de respuestas: "creo que la clase es entretenida y me gusta mucho la manera de explicar los ritmos, paso por paso, especialmente para mí que casi no sé nada de baile". Otra alumna indicó, "he aprendido bastante, muchas vueltas, a seguir al hombre, ritmos que no conocía”. Estos datos coinciden con lo mencionado por Rial (2012). Ella opina que un proceso de progresión es importante porque le permite a la persona instructora tener distintos niveles de aprendizaje en una misma sesión. Según Rial, una progresión consiste en "el conjunto de pasos y movimientos, que se utilizan para llegar al movimiento final con la menor dificultad posible" (p. 6). Además, menciona que una buena progresión se da "cuando se pasa de lo sencillo a lo difícil, de forma eficaz, continua y fluida conservando la intensidad en todo el proceso" (p. 6).

Por lo tanto, con la información analizada, se encontró que los contenidos del curso se fueron desarrollando de manera progresiva, enseñando los pasos y vueltas, uno a uno, y de lo más básico a lo más complejo. Esta característica se vio reflejada en las notas de campo, al evidenciar como al inicio del curso las vueltas, pasos y rutinas eran sencillos, pero, a medida que el ciclo lectivo avanzó, se fueron agregando variantes y aspectos más complejos en las figuras, así como "adornos" a los movimientos y se usaron desplazamientos y pasos más complicados en las rutinas de ejercicios bailables.

Existen ciertas acciones útiles para la enseñanza de la danza. Entre ellas: 1- seleccionar la danza según el nivel del estudiantado, 2- comentar acerca de la procedencia o historia de la danza que se va a enseñar, 3- aprender los pasos básicos, 4- escuchar la música, 5- explorar y sentir la música al realizar movimientos con diferentes segmentos o con todo el cuerpo, 6- pulsar la pieza, 7- practicar las figuras sin música, y 8- practicar las figuras con música (Delgado, 1995).

De acuerdo con las notas de campo y videos, se halló que la acción 1 se cumplió, porque se observó que los bailes enseñados iban acordes con el nivel del estudiantado. Por ejemplo, la profesora, desde el primer grupo focal, indicó, "en todos los ritmos que veamos se empezará por lo más básico y, a medida que avancemos, se irán viendo más pasos y vueltas. Además, empezaremos con un ritmo que no es tan complejo, como el merengue y, luego, otros ritmos cuyos pasos son un poco más complicados, como el swing criollo. Y también dependiendo del avance del grupo, se contemplará la posibilidad de ver otros ritmos como vals, tango o bolero criollo".

La acción 2 también se llevó a cabo porque tanto la profesora como el asistente, antes de enseñar un nuevo género, explicaban brevemente la historia y procedencia de ese ritmo. En cuanto a la acción 3 (aprender pasos básicos) también se realizó con cada género que se enseñó. Se observó como la profesora o el asistente, al empezar algún ritmo, primero enseñaban los pasos básicos.

Con respecto a las acciones 4 y 5, relacionados con escuchar y explorar la música, se realizaron parcialmente. Sólo en algunas actividades de calentamiento, el estudiantado oía la música y se movía según el ritmo, improvisando distintos movimientos.

En lo que respecta a la acción 6 (pulsar la pieza), este no se desarrolló, porque la cantidad de clases era poca, por lo que el personal docente enfatizó en el aprendizaje y práctica de las figuras. De hecho, esta circunstancia la señaló la profesora en el primer grupo focal, cuando mencionó "vamos a aprovechar al máximo el tiempo, porque el semestre se va muy rápido y a pesar que se enseñan bastantes ritmos, siempre hace falta tiempo para ver más”. 
Por otra parte, las acciones 7 y 8, que se referían a practicar las figuras primero sin música y luego con música, se hicieron evidentes en cada una de las vueltas enseñadas por la profesora o el asistente, de acuerdo con los datos de las observaciones, videos y reportes del asistente.

\section{o Considerar el estilo de baile.}

En la clase, el repaso y la enseñanza de figuras contemplaron que las parejas cambiaran para bailar con diferentes personas. La mecánica que se usó para el cambio de parejas fue hacer dos círculos concéntricos, uno de hombres y otro de mujeres. Al finalizar cada pieza, las mujeres u hombres (dependiendo de la indicación de la profesora) rotaban un espacio hacia su derecha. Sin embargo, no siempre quedaban pares. Entonces, por ejemplo, si dos mujeres no tenían pareja, los hombres rotaban dos espacios hacia su derecha. Este mecanismo funcionó en la mayoría de las ocasiones. No obstante, la profesora y el asistente tuvieron que reforzar la supervisión de la rotación, porque, a veces, las personas irrespetaron el orden establecido para el cambio de parejas, según el comentario que dieron algunas alumnas al personal docente. Esta situación provocó que, en varias ocasiones se bailara con un menor número de personas, debido a que algunos participantes repetían pareja. Sin embargo, según la docente esta circunstancia de repetir con quien se bailaba, se hubiera visto incrementada si no se hubiera formado el doble círculo y se hubiera dejado libre la escogencia de pareja. Según la profesora, "si no se hacen círculos, los estudiantes no se relacionarían tanto, y se haría una mayor cantidad de subgrupos" y se "impediría conocer mejor a los demás compañeros, al mismo tiempo que se practicarían menos estilos de baile”.

Los bailes de salón ofrecen una amplia gama de posibilidades y estilos (Padilla y Zurdo, 2002). De acuerdo con Guadalupe Delgado (comunicación personal, 29 abril 2003), el estilo de baile se refiere a la forma en que cada persona baila determinado ritmo. Al respecto, Fernández, Ros y Vera (1998) señalan que uno de los elementos necesarios en la danza es la música, la cual es igual para todas las personas. No obstante, lo que se expresa mediante la música es diferente para cada persona y depende de lo que se quiera y se pueda demostrar con el cuerpo.

Por otra parte, Salomó (1996) considera que la enseñanza del baile debe orientarse para que el estudiantado desarrolle su propio estilo, por medio de la creación de sus propios movimientos y desplazamientos, y el desarrollo de la creatividad. Aunque se realicen los mismos pasos y figuras, cada estudiante tiene una personalidad diferente que determina la actitud y la expresividad hacia el baile. Esta situación de procurar que el alumnado desarrolle y practique su propio estilo de baile fue fomentada por la profesora al inicio del semestre, en el primer grupo focal. Ella insistió en la importancia de rotar parejas y no bailar con una misma persona toda la lección, para tener la oportunidad de conocer distintos estilos de baile.

\section{- Participación activa y tipo de evaluación durante la lección.}

Al analizar la información de las observaciones y de los grupos focales, se rescataron dos factores que, al parecer, influyeron en el agrado de la metodología del curso: primero, el evitar momentos pasivos y, segundo, el tipo de evaluación. En el grupo focal, una estudiante indicó "casi no tuvimos que esperar turno, por lo general siempre teníamos pareja y esto ayudó para que no fuera aburrido”. A lo cual, la profesora respondió, “tiene mucha razón porque en otros 
grupos, donde el faltante de hombres o mujeres es mayor, genera menos tiempo de práctica para los estudiantes y se puede volver tedioso para ellos".

En cuanto al segundo factor acerca de la evaluación, una estudiante manifestó, "estoy cansada de que me hagan exámenes y quiz, creo que tiene que haber otra forma de evaluar y creo que en este curso esto me ha ayudado a sentirme cómoda y sin presión, por eso lo disfruto más". La profesora opinó que la evaluación del curso permite que "el estudiantado no se preocupe por estar dentro de cierto parámetro en cuanto a su técnica, sino lo que debe hacer es asistir y participar en la lección y si esto sucede con estudiantes que matricularon el curso porque realmente lo que querían era aprender y practicar, o es uno de los que disfruta la clase, no va a sentirse mal por asistir al curso, al contrario se verá realizado, contento y obtendrá gran cantidad de beneficios”. Según Salazar (2007), la recreación se caracteriza por incluir actividades de libre elección y participación que son parte de la educación no formal, producen gozo y causan desarrollo integral en aquellas personas que las practican. De cierta manera, el curso de baile popular llegó a parecerse a una actividad recreativa, para aquellas personas que realmente lo desearon matricular y que durante su práctica lo disfrutaron, aunque dicha asignatura formaba parte de la educación formal y su participación en clase fuera obligatoria. Al respecto, Salazar (1995) indica que cuando se enseña danza/baile con una dimensión recreativa, los grupos se vuelven más exitosos porque disfrutan lo que hacen. Por lo tanto, el deseo de participar en la clase y disfrutar haciendo, le facilitó a la población estudiantil cumplir con el criterio de evaluación del curso, que consistía en una participación activa durante la lección y una asistencia casi del 100\% durante el semestre.

\section{- Estilos de enseñanza.}

Luego de analizar las observaciones, videos, y reportes escritos del asistente, se detectó que entre los métodos más utilizados para la enseñanza del baile popular durante el curso se hallaban el comando directo y la resolución de problemas.

\section{- Comando directo.}

El comando directo es el método en el que la persona docente toma todas las decisiones y la persona aprendiz acata las órdenes cuando se le indica (Moston, 1986). Este método se usó mucho para la enseñanza de las distintas vueltas de baile y para las rutinas de ejercicios bailables. Con base en la información recabada con las técnicas cualitativas, se observó que tanto la profesora y como el asistente enseñaban la figura al mismo tiempo que el alumnado iba realizando la vuelta. En cuanto a las rutinas, se evidenció cómo la profesora o el asistente realizaban un paso y, casi al mismo tiempo, el estudiantado lo hacía, para copiar la secuencia establecida. El uso de este estilo permite aprovechar mejor el tiempo, pero restringe la creatividad e independencia del alumnado (Moston, 1986). Sin embargo, esta debilidad se vio solventada al usar otro estilo de enseñanza como el de resolución de problemas. 


\section{- Resolución de problemas.}

Romero (2010) menciona que generar actividades en las que se desarrolle la improvisación y en las que no se busque una secuencia de pasos, también es útil porque estimula en el estudiantado la creatividad, la imaginación, la originalidad y la práctica de pasos y vueltas, de una manera más libre y espontánea. Este señalamiento coincide con el estilo de enseñanza de resolución de problemas, propuesto por Moston en 1986. En este estilo, la persona docente plantea un problema al estudiantado y estos intentan resolverlo. Con esta acción se fomenta la creatividad e independencia. Además, el papel del personal docente consiste en retroalimentar y orientar al alumnado (Moston, 1986).

Considerando los hallazgos en este estudio, se encontró que el estilo de resolución de problemas fue utilizado en algunas actividades de calentamiento, en las que el alumnado tuvo que crear movimientos para que las demás personas participantes los repitieran. También, se evidenció cuando la profesora asignó a la población estudiantil realizar una coreografía. Para esta tarea, el alumnado tuvo el tiempo de una lección y media. La coreografía fue presentada en la última clase del semestre, en el cierre del curso. En este caso, la profesora y el asistente brindaron apoyo y orientación, pero la creación, la escogencia de la música, de los pasos, de las vueltas, del vestuario y duración de la puesta en escena, quedaron a criterio y creatividad de la población estudiantil.

\section{Labor del personal docente durante la lección de baile popular}

Otros aspectos por tener en cuenta entre los resultados encontrados corresponden a la actitud y al desenvolvimiento de la profesora y del asistente durante la lección y cómo su labor influyó en el aprendizaje del alumnado.

\section{- Planeación, explicación, demostración y relación con el grupo.}

De acuerdo con las notas de campo y reportes escritos del asistente, las lecciones se desarrollaron con un planeamiento que fomentaba la enseñanza progresiva, tanto de las figuras como de las rutinas de ejercicios. Además, según los datos obtenidos en las tarjetas de respuestas, no se encontró ningún comentario que presentara insatisfacción por el curso o por la labor de la docente. Por el contrario, hubo agrado por la manera en que se enseñaba. Por ejemplo, entre las respuestas escritas en las tarjetas se halló, "el curso está muy bien, estoy aprendiendo mucho", "bastante provechoso, la clase se presta para el aprendizaje general", "los profes enseñan bien, estoy aprendiendo mucho, a pesar de que no sabía bailar".

Sin embargo, dos alumnos señalaron en las tarjetas de respuesta que el asistente tenía que tener cuidado con las explicaciones porque, a veces, se enredaba. De acuerdo con las notas de campo, esta situación fue detectada por la profesora quien acudía a ayudarlo, intervenía, colaboraba o aclaraba la indicación que había dado el asistente. Además, en el reporte oral y en el escrito del asistente, se detectó la disconformidad del asistente con la manera en cómo había brindado algunas de las explicaciones o demostraciones. Sin embargo, conforme fueron avanzando las lecciones, el asistente reportaba un mayor grado de satisfacción con su 
proceder. Por ejemplo, en uno de los reportes orales, él señaló, “cada vez me siento con más confianza y las vueltas y los pasos me salen mejor”.

Aún tomando en cuenta esta situación, la profesora consideró que "es importante darle oportunidad al estudiantado que hace la práctica profesional, porque esto les permite poner en práctica sus conocimientos y obtener experiencia, ante la realidad de tener un grupo a cargo". Además, a pesar de algunas fallas que cometió el asistente, la profesora cuidó que la intervención de él fuera paulatina, para que, según ella, "el asistente fuera conociendo al grupo y tomando confianza, hasta llegar el momento en que solito realizara toda la clase”. Por ejemplo, en las notas de campo y reportes, se encontró que el asistente se hizo cargo, primero, de una charla de información, luego de un calentamiento, después del calentamiento y la enseñanza de algunas figuras. Posteriormente, se le asignó la enseñanza de una rutina de ejercicios bailables, y así sucesivamente hasta el día en que se encargó de la lección completa.

Por otra parte, es necesario tener en cuenta que la manera en que se imparte la lección es importante y depende de varios aspectos. Según Salazar (1995), para la enseñanza del baile se deben tener en cuenta varios factores: (1) la preparación del docente, que incluye el conocimiento, las destrezas y la actitud que éste posea, (2) la habilidad para planear; es decir, tener en cuenta los objetivos, una enseñanza progresiva con buena organización y la evaluación, (3) la habilidad para presentar los pasos, para ello se debe tener en cuenta la voz, las explicaciones y las demostraciones claras, para facilitar el control de grupo, y (4) la sensibilidad a la respuesta del grupo, o sea, la capacidad de adaptarse, ajustarse, analizar y socializar con el grupo que se tenga a cargo. Varios de estos factores fueron confirmados que se llevaron a cabo por el personal docente, por medio de los comentarios de la población estudiantil en las tarjetas de respuestas. Algunos de ellos señalaron, "he aprendido mucho", "buenisimo", "se aprende bastante, las clases bien estructuradas", "el curso me ha gustado bastante, la manera de impartir la clase me parece adecuada", "aprendi bastante con estas clases, a pesar de no haber bailado nunca antes", "las instrucciones de parte de los profes son claras, me siento complacida con el curso, pues he aprendido bastante de los ritmos impartidos durante la clase”.

Además, con respecto a la relación con el grupo, se encontró tanto en las notas de campo como en los videos que, en varias ocasiones, la enseñanza de las figuras de baile se realizaron de manera amena, incluso haciendo algún chiste por parte de la docente, a lo cual el estudiantado respondió con risas.

\section{- Supervisión y retroalimentación.}

Otra característica importante que se rescató de las notas de campo fue el reforzamiento y la retroalimentación positiva que la docente y el asistente brindaron al alumnado. Por ejemplo, por medio del video se evidenciaron frases de la profesora que alentaban al estudiante como "muy bien", "te salió excelente", "todo les salió super bien, solo cuiden este detallito para que les salga mejor, pero en general está genial”. Estas frases positivas y que ayudan a corregir la técnica sin insultar o irrespetar a quienes las reciben, provocaron motivación en el estudiante que, a su vez, agradeció con palabras como "ok, profe, muchas gracias", "pura vida”, "ah, ok, con razón no me salia, muchas gracias”. Además, la profesora insistió al asistente en que 
reforzara al estudiantado, que se acercara a las parejas y rescatara lo bueno que estaban haciendo o el progreso que estaban teniendo. Que no solo se acercara a una pareja para corregir sino que también lo hiciera para reconocer el esfuerzo y algún logro.

Esta retroalimentación es alentada por Chacón y Hernández (2004), quienes indican que la persona instructora debe ser entusiasta, ayudar al estudiante a superar las dificultades, el cansancio y a concentrarse de manera relajada. Debe ser sensible a las necesidades y problemas del alumnado y dar apoyo en caso de inseguridad.

\section{Relaciones interpersonales entre estudiantes durante el curso de baile popular}

Un aspecto que se llegó a analizar, por medio de las diferentes técnicas cualitativas, fue las relaciones interpersonales del alumnado. Fue interesante observar cómo interactuaban las distintas personas y examinar si las relaciones interpersonales entre la población estudiantil eran agradables y facilitadas gracias a la participación en las actividades del curso de baile popular.

\section{- Ambiente ameno.}

Desde la primera clase se observó que las actividades desarrolladas por la profesora pretendían fomentar las relaciones interpersonales entre el alumnado. Por ejemplo, el primer día de clases se realizaron varias actividades de integración y rompe-hielo que facilitaron la interacción entre las personas participantes. Al respecto, la profesora señaló "las actividades que vamos a realizar hoy están programadas para que ustedes se conozcan, compartan, a la vez trabajen la confianza, el respeto y la cooperación".

En general, durante las lecciones, se observó un ambiente ameno en la clase, en el cual el estudiantado se llevaba bien y se observaba entusiasta con el curso. En el segundo grupo focal, la profesora mencionó, "las personas que integran los grupos de baile popular, por lo general son muy alegres y simpáticas". Una estudiante también comentó, "diay, profe como usted mencionó al inicio del curso, el baile es una actividad muy sociable, que permite compartir mucho y hablar con todos". Otra alumna agregó, "yo creo se debe a que todos tenemos una edad parecida y eso ayuda a llevarnos bien". Otra señaló, "yo creo más bien que se debe a que a todos nos une el gusto por el baile". A lo que la profesora respondió, "en realidad, todos estos factores tienen que ver. Y sí, el baile es muy sociable y en este caso se necesita alguien para bailar, por lo que involucra comunicación y estar cerca de otra persona". Estas apreciaciones coinciden con lo establecido por Padilla y Zurdo (2002), acerca de que los bailes de salón, también conocidos como danza social, se caracterizan por el trabajo en parejas, por el aprendizaje ameno, y porque se puede practicar con personas de todas las edades.

\section{- Promoción de valores.}

En la mayoría de los comentarios escritos en las tarjetas de respuestas, el estudiantado señaló que se sentía cómodo con el grupo y consideraba que las personas compañeras eran amables, decentes, humildes y simpáticas. Incluso, un alumno mencionó, "uno se siente bien con el compañerismo que se da en el grupo y hasta logra aprender más fácil”. Este 
compañerismo también se observó en los videos, en los cuales se veía como constantemente aquellas personas que sabían más o les era más fácil el aprendizaje, ayudaban a aquellas que les costaba un poco más. También, las personas más avanzadas enseñaban otras vueltas, mientras practicaban las que el asistente o la profesora habían enseñado.

Una última actividad que generó que la población estudiantil se uniera más, trabajara en equipo y se beneficiara individual y grupalmente fue la creación y la presentación de la coreografía. El día de las presentaciones, la profesora procuró que todo transcurriera de una manera lúdica. Por ejemplo, les pidió que le pusieran un nombre al grupo, hizo una rifa para determinar el momento en el que se iba a presentar cada grupo, los anunció como si fuera una competencia real e inventó frases, como por ejemplo: "con ustedes desde Puerto Rico, el grupo número 2, llamado Los chicos del merengue. Démosles un fuerte aplauso".

La experiencia de la presentación de la coreografía, según los comentarios del alumnado (plasmados en las notas de campo), fue muy gratificante porque les permitió poner en práctica lo aprendido durante el curso. Aunque señalaron que, a veces, fue difícil ponerse de acuerdo con los pares, pero que en la mayoría de las ocasiones hubo consenso, apoyo, propuestas y comunicación. Uno de los alumnos indicó, "para mi fue toda una experiencia, jamás había bailado y mucho menos presentado ante un público. Me siento feliz por haberlo logrado”. Otra señaló, "la verdad es que era una tarea difícil, pero lo bueno fue que todos ponían ideas y proponían cosas, y la coreo se fue armando". Al final de las presentaciones la docente manifestó un alto grado de satisfacción por la labor y el esfuerzo realizado por sus estudiantes.

Por lo tanto, se rescata cómo este tipo de actividad, al ser creada por el mismo estudiantado, fortalece a las personas de manera individual, al fomentar la auto-superación y la confianza y, grupalmente, se promueven valores como el respeto, el trabajo en equipo, la comunicación y la tolerancia. Estos hallazgos se evidenciaron en las reacciones del alumnado, al que en todo momento se le notó alegre, motivado, participativo. Aplaudían, chiflaban y decían frases de aliento como, "eso chicos", "vamos, vamos". Incluso, cuando la profesora les consultó que cómo se sentían, un alumno indicó, "estuve nervioso, pero al final creo que todo salió muy bien". Otro estudiante dijo "me gustó mucho, yo no sabía bailar y ahora me siento con más confianza, por lo menos para no quedarme sentado en una fiesta”.

Estos resultados coinciden con los encontrados en el estudio de Molina (2009), en el cual un grupo de adolescentes identificaron varios beneficios físicos, emocionales y sociales, luego de participar en un programa de danza. De acuerdo con Molina, además de la funcionalidad física y la reducción del estrés, las personas participantes mencionaron sentimientos de felicidad, amistad, satisfacción, trabajo en equipo y cooperación, gracias a la participación en el programa de danza.

\section{- Normas de higiene.}

No obstante, una parte del estudiantado indicó que era importante que las personas compañeras recordaran las medidas de higiene que se mencionaron al inicio del curso, porque algunos no las cumplían. La profesora, al inicio del curso, señaló varias medidas de higiene necesarias de respetar, tanto en asuntos de aliento como de sudor, debido a que el 
tipo de actividad física era intensa y se debía brindar comodidad a la persona con la que iba a bailar. Esta norma la volvió a reforzar el asistente, a solicitud de la profesora, por medio de una charla de etiqueta en el baile. Al respecto, en el artículo de Etiqueta y buenas maneras en el baile (s.f.), se indica que se debe cuidar la higiene personal y controlar el cabello largo. Cuando se respetan estas normas, se genera comodidad para la pareja con la cual se baila, y se permiten que, indirectamente, las relaciones entre las personas sean llevaderas y cómodas. A la vez, que fomentan valores como el respeto y la empatía por los demás. Estas normas coinciden con lo señalado por Gálvez y López (2007), en el sentido de que la danza fomenta el contacto social con los demás.

Por lo tanto, según la información analizada en este apartado, se puede considerar que las actividades llevadas a cabo en el curso de baile popular estimularon las relaciones interpersonales, porque se vieron facilitadas por un ambiente ameno y permitieron el fomento de valores y de las normas de higiene. Los hallazgos también coinciden con Valverde (2002). Él señala que la práctica de la danza incrementa las relaciones interpersonales.

\section{Implicaciones de los resultados y recomendaciones}

De acuerdo con los resultados, se debe tener presente que las clases de actividad deportiva o de educación física constituyen la oportunidad para que el personal docente haga conciencia en la población estudiantil que, a pesar de la falta de tiempo, es de vital importancia realizar actividad física, por los beneficios que provee a la salud. El baile popular es una opción divertida para hacer ejercicio, cuidar la salud y fomentar las relaciones interpersonales. Además, las lecciones deben tener el objetivo de enseñar y mejorar destrezas que le sean útiles a las personas para poner en práctica durante su vida cotidiana y así conformar un estilo más saludable de vida. Por esa razón es importante generar espacios agradables para que el estudiantado se ejercite al mismo tiempo que aprende, se divierte y comparte con otras personas.

Para que las lecciones cumplan con los objetivos anteriormente mencionados es necesario procurar que las actividades sean variadas, atractivas, con una estructura planificada, con procesos metodológicos óptimos y estilos de enseñanza variados. Las clases deben contemplar en el caso del baile popular, variedad de calentamientos, de actividades rítmicas, de dinámicas que fomenten el estilo de baile, el uso del ritmo y promover la participación activa que conlleve a la autoevaluación.

Sumado a lo anterior, se debe concientizar a la población estudiantil que el baile es una actividad social, que permite y fomenta la convivencia y la comunicación, que procura la ayuda mutua, el compañerismo, la compresión y la tolerancia, pero que, a la vez, demanda respeto y empatía por la persona con quien se está bailando. También es importante que, al enseñar, el personal docente domine los contenidos, sea cordial con la población estudiantil, atienda sus necesidades y, a la vez, muestre elocuencia y versatilidad a la hora de instruir.

Los hallazgos de esta investigación quedan al servicio de la población docente, con el fin de que sean transferibles, según la realidad de cada contexto educativo. 


\section{Conclusiones}

Con base en los objetivos planteados en este estudio, se concluye que la metodología usada en el curso de Baile Popular y la labor docente influyeron tanto en el aprendizaje como en el esparcimiento del alumnado. Estos logros se evidenciaron por medio de las acciones y las actividades desarrolladas en el curso, las cuales promovieron que las destrezas dancísticas del alumnado mejoraran. Este hecho se detectó al observar el desenvolvimiento del estudiantado, desde el momento en que inició el proceso hasta la última lección. A la vez, la metodología y la labor docente permitieron que se generara una sensación de gozo y satisfacción en las personas participantes durante las clases. Además, se detectó que el trabajo realizado en las clases influyó en las relaciones interpersonales del estudiantado y se evidenció en el fomento de valores, normas de higiene, así como en la comunicación y el trabajo en equipo.

\section{Referencias}

Artavia, J. (2011). Juegos e interacción social en los recreos y su relación con los comportamientos violentos: El caso de las y los estudiantes de VI año de una escuela de la Dirección Regional de Educación Occidente (Tesis de doctorado sin publicar). Universidad Estatal a Distancia, San José, Costa Rica.

Bernal, J. y Calvo, M. (2000). Didáctica de la música. La expresión musical en la Educación Infantil. Málaga: Aljibe.

Castillo, L., Alvarado, A. y Sánchez, M. (2006). Enfermedad cardiovascular en Costa Rica. Revista Costarricense de Salud Pública, 15(28), 3-16.

Chacón, Y. (2003). Antología del curso EF 7012 Movimientos y ritmos básicos. Manuscrito sin publicar, Escuela de Educación Física y Deportes, Universidad de Costa Rica, San José, Costa Rica.

Chacón, Y. y Hernández, J. (2004). Antología del curso danza aeróbica. Manuscrito sin publicar, Escuela de Educación Física y Deportes, Universidad de Costa Rica, San José, Costa Rica.

Colás, M. y Buendía, L. (1994). Investigación educativa. Sevilla: Ediciones Alfar.

Delgado, G. (1995). Propuesta metodológica para la enseñanza de las danzas tradicionales y populares. Memoria III Congreso Nacional de Cultura Popular (pp.107-121). Costa Rica: la autora.

Etiqueta y buenas maneras en el baile. (s.f.). Etiqueta y buenas maneras en el baile. Recuperado de http://www.yoquierobailar.com/modules.php?name=News\&file=article\&s id $=58 \&$ mode $=\&$ order $=0 \&$ thold $=0$ 
Fernández, J., Ros, N., y Vera, A. (1998). Educación física. Libro cuaderno de patio $4^{\circ}$ E.S. España: Editorial Pila Teleña, S.L

Fubini, E. (1998). La estética musical desde la antigüedad hasta el siglo XX. Madrid: Alianza músical.

Gálvez, J. y López, M. (2007). Locos por el baile. Recuperado de http://dialnet.unirioja.es

Garber, C., Blissmer, B., Deschenes, M., Franklin, B., Lamonte, M., Lee, I., Nieman, D., y Swain, D. (2011). Quantity and quality of exercise for developing and maintaining cardiorespiratory, musculoskeletal, and neuromotor fitness in apparently healthy adults: Guidance for prescribing exercise. Medicine \& Science in Sports \& Exercise, 43(7), 13341359. DOI: http://dx.doi.org/10.1249/MSS.0b013e318213fefb

García, S. (1979). Evolución de la actividad deportiva en la Universidad de Costa Rica, desde 1957 hasta 1978 inclusive. Manuscrito sin publicar, Escuela de Educación Física y Deportes, Universidad de Costa Rica, San José, Costa Rica.

Garnier, L. (2009). Proyecto de ética, estética y ciudadanía. Programas de estudio de educación física. Versión preliminar. Manuscrito sin publicar, Ministerio de Educación Pública, San José, Costa Rica.

Goetz, J. y LeCompte, M. (1988). Etnografía y diseño cualitativo en investigación educativa. España: Ediciones Morata, S. A.

Gremion, G. (2005). Is stretching for sports performance still useful? A review of the literature. Revue Medicale de la Suisse, 1(28), 1830-1834.

Martin, M. (1999). Aerobic y fitness. Fundamentos y principios básicos. Madrid: Ed. SM. SL.

Molina, M. (2009). La participación en un programa de danza contemporánea dentro de un marco de aprendizaje constructivista como opción recreativa de personas adolescentes pertenecientes al grupo juvenil ICTHUS de la Primera Iglesia Bautista de Heredia (Tesis de maestría sin publicar). Universidad de Costa Rica, San José, Costa Rica.

Moston, M. (1986). Enseñanza de la educación física. Estados Unidos: Macmillan Publishing Company.

Muñoz, D. (2009). El calentamiento en educación física. Fundamentos, tipos y funciones. Sesión práctica. EFDeportes.com, Revista Digital, 13(129), 1. Buenos Aires. Recuperado de http://www.efdeportes.com/efd129/el-calentamiento-en-educacion-fisica.htm 
Padilla, C. y Zurdo, R. (2002). Danza: Una oferta de ocio para adolescentes. En Virtual III Congreso Internacional de Educación Física: Educación Física, Ocio y Recreación. Cádiz. Recuperado de http://www.expresiva.org/files/PDF_Articulos/X006_Danza_oferta_ocio.pdf

Pérez, B., Robinson, P., y Herlong, K. (2010). Instructor training manual basic steps level 1. Florida: Zumba Fitness, LLC.

Reyes, J. (2005). La agresividad y violencia en los juegos de los niños durante el recreo escolar (Tesis de maestría sin publicar). Universidad Pedagógica Nacional, Chihuahua, Méjico.

Rial, T. (2012). Revisión de los métodos de construcción coreográficos en actividades dirigidas con soporte musical. EFDeportes.com, Revista Digital, 16(165), 1-10. Buenos Aires. Recuperado de http://www.efdeportes.com/efd165/metodos-de-construccion-coreograficos.htm

Rodríguez, A., Villalobos, T. y Mac Donald, J. (2006). Después de la línea del tren. Costa Rica: Universidad Nacional, Costa Rica.

Romero, C. (2009a). Actividades rítmicas para una lección de educación física, bajo una metodología constructivista. En Escuela de Educación Física y Deportes, Universidad de Costa Rica, Memoria del XVI Simposio de Ciencias del Deporte y la Salud. San José, Costa Rica: la autora.

Romero, C. (2009b). Barreras que impiden la participación en el baile popular en estudiantes de grupos de Actividad Deportiva de Baile Popular Principiante Mixto de la Universidad de Costa Rica. En Escuela de Educación Física y Deportes, Universidad de Costa Rica, Memoria del XVI Simposio de Ciencias del Deporte y la Salud. San José, Costa Rica: la autora.

Romero, C. (2010). Enseñanza del baile popular a través del juego. En Universidad Nacional Autónoma de Nicaragua, Memoria del V Congreso Centroamericano de Educación Física, Deportes y Recreación. Managua, Nicaragua: la autora.

Romero, C. (2012). Programa del curso baile popular principiante mixto. Manuscrito sin publicar, Escuela de Educación Física y Deportes, Universidad de Costa Rica, San José, Costa Rica.

Rubio, I. (s.f.). El juego como medio de calentamiento en la educación física y en el entrenamiento. Recuperado de http://www.inefi.gob.do/LinkClick.aspx?fileticket=\% $2 \mathrm{FC}$ LunXzDBKc\%3D\&tabid=102\& $\mathrm{mid}=431$

Salazar, C. (1995). Folleto de teoría y coreografías de danzas y bailes internacionales. Manuscrito sin publicar, Escuela de Educación Física y Deportes, Universidad de Costa Rica, San José, Costa Rica. 
Salazar, C. (2007). Recreación. San José, Costa Rica: Editorial de la Universidad de Costa Rica.

Salomó, C. (1996). Unidades didácticas para secundaria III. Bailes de salón. España: Editorial INDE.

Sandin, E. (2003). Investigación cualitativa en educación. Fundamentos y tradiciones. España: Mcgraw-hill/interamericana de España, S. A. U.

Quiñónez, Y. (2006). Las actividades rítmicas. Recuperado de http://actividadesritmicas. blogspot.com/

Valverde, O. (2002). Toques para estar en todas: Módulo socioeducativo para el fortalecimiento de conductas protectoras y la prevención de conductas de riesgo en los y las adolescentes. Costa Rica: CCSS-PAIA.

Zavatto, L. y Gabbei, R. (2008). The real dance revolution: How to make dance meaningful for all students. A Journal for Physical and Sport Educators, 21(5), 25. 